PHYSICAL REVIEW D 96, 109902(E) (2017)

\title{
Erratum: Observational constraints on decoupled hidden sectors [Phys. Rev. D 94, 063506 (2016)]
}

Matti Heikinheimo, Tommi Tenkanen, Kimmo Tuominen, and Ville Vaskonen (Received 3 November 2017; published 16 November 2017)

DOI: 10.1103/PhysRevD.96.109902

We have discovered a typographical error affecting Eqs. (10) and (15). The correct form of Eq. (10) is

$$
x_{\mathrm{D}}^{\mathrm{FO}}=\frac{1}{3} \log \left(\left(\frac{1}{2 \pi}\right)^{\frac{9}{2}} \frac{\xi^{2} \lambda_{\mathrm{s}}^{4} M_{\mathrm{P}}}{1.66 \sqrt{g_{*}} m_{\mathrm{s}}\left(x_{\mathrm{D}}^{\mathrm{FO}}\right)^{\frac{5}{2}}}\right),
$$

and the correct form of Eq. (15) is

$$
x_{\mathrm{D}}^{\mathrm{FO}}=\frac{1}{3} \log \left(\left(\frac{1}{2 \pi}\right)^{\frac{9}{2}} \frac{\xi^{2} g^{8} M_{\mathrm{P}} m_{\psi}^{9}}{1.66 \sqrt{g_{*}} m_{\mathrm{s}}^{10}\left(x_{\mathrm{D}}^{\mathrm{FO}}\right)^{\frac{5}{2}}}\right) .
$$

Our analysis and results remain unchanged. 